\section{Prevalence of falls and associated factors in community-dwelling older Brazilians: a systematic review and meta-analysis}

\author{
Prevalência de quedas e fatores associados em \\ uma amostra comunitária de idosos brasileiros: \\ uma revisão sistemática e meta-análise
}

\section{Prevalencia de caídas y factores asociados en ancianos brasileños residentes en una comunidad: revisión sistemática y metaanálisis}

José Elias Filho 1 Wyngrid Porfirio Borel 1 Juliano Bergamaschine Mata Diz 1 Alexandre Wesley Carvalho Barbosa 2 Raquel Rodrigues Britto 1 Diogo Carvalho Felício 1

doi: 10.1590/0102-311X00115718

\begin{abstract}
Falls determine huge epidemiological, clinical, and economic burden in the older population worldwide, presenting high odds of severe disability. The present study aimed to estimate the prevalence of falls and associated factors in older Brazilians using a systematic review with meta-analysis. Searches were performed in SciELO, PubMed, LILACS, Web of Science, Scopus and PsycINFO databases with no date or language restrictions. Studies on community-dwelling older persons aged $\geq 60$ years from both sexes and with a sample size of $\geq 300$ participants included. Exclusion criteria were studies conducted specifically for older adults diagnosed with chronic disabling diseases that predispose them to falls. Risk of bias of included studies was assessed using a critical appraisal tool focusing on prevalence designs. A random-effects meta-analysis was used to pool the prevalence of falls across studies. Exploratory analysis was conducted examining subgroup estimates, prevalence ratios and metaregression. Thirty-seven studies involving 58,597 participants were included. Twelve-month prevalence of falls was 27\% (95\%CI: 24.3-30.0), with significantly higher estimates in female than male ( $P R=1.57$; 95\%CI: 1.32-1.86), in age group $\geq 80$ years than age group 60-69 years $(P R=1.46$; 95\% CI: 1.15 1.84), and in participants from the Central region than participants from the South region $(P R=1.36$; 95\%CI: 1.10-1.69) of Brazil. Risk of bias scores did not impact heterogeneity in the 12-month meta-analysis. These estimates strongly support evidence-based public interventions to prevent falls in older Brazilians, especially in women and the oldest-old population.
\end{abstract}

Accidental Falls; Aged; Prevalence

\author{
Correspondence \\ J. Elias Filho \\ Universidade Federal de Juiz de Fora. \\ Av. Eugennio do Nascimento s/n, Juiz de Fora, $M G$ \\ 36038-330, Brasil. \\ joseeliasfilho@yahoo.com.br \\ 1 Universidade Federal de Juiz de Fora, Juiz de Fora, Brasil. \\ 2 Universidade Federal de Juiz de Fora, Governador Valadares, \\ Brasil.
}




\section{Introduction}

Falls have multifactor causes in old age and may occur due to decreased muscle mass and strength, reduced mobility, flexibility, visual acuity, psychological aspects such as depression and fear of falling, cognitive decline, gait changes, postural disorders and body imbalance 1,2 . In addition, the literature highlights extrinsic factors such as inadequate lighting, type of flooring, roadblocks and uneven terrain 3,4 .

According to the most recent public data collected over a five-year period, the number of falls in older Brazilians treated at the Brazilian Unified National Health System (SUS) facilities and subsequently hospitalized was 399,681, costing the Ministry of Health a total of BRL 464,874,275.91 $(\approx$ USD 138,002,773.85) 5. A study conducted in Bahia State in 2014, characterized hospitalizations and the cost of falls in older persons and reported a total of 4,851 hospitalizations, an average stay of seven days, and total costs of BRL 5,842,576.52 ( $\approx$ USD 1,734,498.68) 6 .

The need to adopt educational and preventive measures aimed at health promotion is recognized in Brazil. Multi-sector public policies geared to the care of the older population have been recommended for several decades 7,8,9,10. However, policy initiatives should be based on known problems that affect a target population. Studies on fall prevalence in older Brazilians have shown inconsistent results perhaps because it is an extensive country or due to socioeconomic differences between regions. The prevalence was 53.6\% in the city of Natal (Rio Grande do Norte State) 11, 32.1\% in the city of Juiz de Fora (Minas Gerais State) 12, 16.1\% in the city of São Paulo 13, and 34.8\% in South and Northeast regions 14 .

A robust study with a representative sample assessed the prevalence of falls in the urban areas of 100 municipalities in 23 Brazilian States, including 6,616 older participants aged $\geq 60$ years 15 . The municipalities were selected based on data from the official 2000 census. The prevalence of falls was estimated at $27.6 \% 15$. Scientific evidence on falls is essential to guide clinical practice, research and health policies.

From the authors' knowledge, there is no systematic review on the prevalence of falls in older adults living in Brazil. As such, the aim of the present study was to conduct a large-scale systematic review and meta-analysis by estimating the prevalence of falls and examining associated factors in community-dwelling Brazilian people aged 60 years and older.

\section{Materials and methods}

\section{Study design and guidelines}

This is a systematic review and meta-analysis. The review protocol was registered at the International Prospective Register of Systematic Reviews (PROSPERO) under number CRD42018092326. All the methodology followed recommendations contained in the Joanna Briggs Institute Reviewers' Manual (The Systematic Review of Prevalence and Incidence Data) 16,17, MOOSE Group (Meta-analysis of Observational Studies in Epidemiology) 18, and Cochrane Collaboration 19. The review was reported according to the PRISMA Checklist (Preferred Reporting Items for Systematic Reviews and Meta-Analyses) 20.

\section{Search strategy}

Searches were conducted until February 2019 in SciELO, PubMed, LILACS, Web of Science, Scopus and PsycINFO databases with no date or language restrictions. Manual searches were also performed in specialized journals and in the reference list of eligible studies. The following descriptors and combinations were used: falls OR falling OR falls, accidental OR accidental AND falls OR fall, accidental OR slip and fall OR fall and slip AND aged OR aging OR older OR elderly OR ancient OR former OR advanced in years OR gray-haired AND Brazil OR Brasil OR Brazilian OR Latin America OR South America OR America (see supplementary data in Appendix 1 - http://cadernos.ensp.fiocruz.br/site/ public_site/arquivo/supl-1-e00115718_8332.pdf). 


\section{Eligibility criteria}

Inclusion criteria were articles on community-dwelling older Brazilians from both sexes, aged 60 years and older whose primary or secondary outcome was point-, period-, or lifetime-prevalence of falls. The following formula was used to estimate sample size:

$$
\mathrm{Z}^{2} * \frac{P(1-P)}{D^{2}}
$$

where $Z$ is the value of $Z$-statistic for the confidence level (95\%), $P$ is the expected prevalence, estimated at $26 \%$, and $D$ is the acceptable error $(0.5 \%) 21$. The expected prevalence was based on an earlier study with older Brazilians 15. Based on this estimate, studies with $\geq 300$ participants were included.

Exclusion criteria were studies conducted specifically with older patients diagnosed with chronic disabling conditions such as Parkinson's disease, stroke, dementia, cancer, diabetes, chronic obstructive pulmonary disease, kidney failure requiring hemodialysis, osteoporosis, rheumatoid arthritis, and osteoarthritis. Previous studies have shown that the morbidities and their coexistence predispose to a greater risk of falling in old age 1,12,13.

\section{Data selection and extraction}

Two independent reviewers (J.E.F. and W.P.B.) screened titles and abstracts of all retrieved citations for checking eligibility. Full-text articles were accessed for review if they met the inclusion criteria. When more than one study reported prevalence of falls using the same sample, only the study with the largest sample was included. The authors of original studies were contacted via e-mail to ask any clarifying questions and/or to obtain unreported data. Information on the study ID, geographical region, sample characteristics, and prevalence estimates by total and by sex were extracted to a standardized form. Disagreements were resolved through consensus.

\section{Risk of bias assessment}

Risk of bias of each included study was assessed independently by two reviewers (J.E.F. and W.P.B.) using a tool developed and validated by Munn et al. 17 for addressing prevalence studies (Critical Appraisal Checklist for Studies Reporting Prevalence Data). This is a 10-item checklist including questions on sample details (e.g. recruitment and size), data collection (e.g. measurements and instruments), and statistical procedures (e.g. methods and analysis) with Yes, Unclear, No, and Not applicable as response options. Yes and No were interpreted as "low" and "high" risk of bias, respectively (see supplementary data in Appendix 2 - http://cadernos.ensp.fiocruz.br/site/public_site/arquivo/supl2-e00115718_2106.pdf).

For the item five (coverage of the identified sample) of the checklist, a response rate of at least $70 \%$ was considered adequate 22, while for the item nine (subgroup identification and analysis), prevalence estimate by at least sex and age group (e.g. 60-69, 70-79, $\geq 80$ years) was considered essential to fulfill such item as low-risk, when appropriate 23 . A third reviewer (D.C.F. or J.B.M.D.) resolved all disagreements. Previous studies have found excellent between-reviewer agreement and reliability to assess risk of bias using the Munn et al. checklist: Cohen's kappa ranging from 0.65 to 0.78 , and intraclass correlation coefficient ranging from 0.83 to $0.9424,25,26$.

\section{Statistical analysis}

Frequency statistics were initially applied to extracted data. Prevalence estimates were obtained using the number of falls (events) and total sample size of each included study. The estimates were expressed in percentages along with the $95 \%$ confidence intervals $(95 \% \mathrm{CI})$. A random-effects meta-analysis was used to pool the prevalence of falls across studies 16,19. Between-studies heterogeneity was assessed with the I2 statistics 16,19. A funnel plot was used to check for publication if more than 10 studies were included in the pooling analysis 27 . 
An exploratory investigation was conducted by using subgroup and meta-regression analyses. Subgroup analysis pooled prevalence estimates by sex, age, and geographical regions. The association between each subgroup stratum and the prevalence estimates were checked using prevalence ratio (PR) based on Poisson regression with robust estimation of variance 28. Meta-regression examined the impact of risk of bias scores on heterogeneity of estimates. Risk of bias was treated as a studylevel covariate. This analysis was conducted using a random-effects regression model 29, along with the restricted maximum likelihood (REML) method to estimate between-study variance 30, and the Knapp-Hartung approach to test the significance of the model 31. Regression coefficient was provided with 95\%CI.

Statistical significance was inferred at a two-sided p-value $<0.05$. Descriptive and inferential analyses were performed with the SPSS, version 19.0 (https://www.ibm.com/). Meta-analyses were performed using the Comprehensive Meta-Analysis software version 3.3.070 (https://www.metaanalysis.com/).

\section{Evidence synthesis}

The overall quality of evidence on the prevalence findings was judged according to the four levels of the GRADE system (Grading of Recommendations Assessment, Development and Evaluation): high-, moderate-, low-, and very-low quality evidence ${ }^{32}$. The high-quality level suggests that further research is very unlikely to change the prevalence estimate, while the very low-quality level indicates that the prevalence estimate is very uncertain. Two reviewers (J.E.F. and J.B.M.D.) independently judged the quality of evidence using GRADE. Disagreements were resolved through consensus-based discussions.

The quality of evidence started from the high-quality level and downgraded by one point if one of the following criteria were present: (a) serious risk of bias: pooled estimates from studies presenting "high-risk" scores in bias assessment, mainly in items 1-3 (sample information) and 5-7 (validity/ reliability of estimates) of the critical appraisal checklist; (b) serious inconsistency: moderate to high heterogeneity of estimates within or among studies ( $\mathrm{I} 2 \geq 40 \%)$; (c) serious indirectness: fall events identified by means other than participants self-reporting 33 (e.g. medical records, proxy information, etc.); and (d) likely publication bias: important asymmetry detected qualitative and quantitatively, or when its analysis was not possible due to small number of included studies (i.e. $\mathrm{k} \leq 10$ ) 27 . Imprecision was not downgraded in the present review because statistical power was ensured for prevalence estimates by including studies with $\geq 300$ participants as detailed in the subheading Eligibility Criteria.

\section{Results}

\section{Descriptive analysis}

Out of the 6,408 studies, 2,210 were excluded because of duplicates. After checking titles and abstracts, 87 articles were read in their entirety. Of these, 37 articles ranging from 2002 to 2019 were included in the review (Figure 1). The 37 included studies represented 25 States and the five geographic regions of Brazil, including a total of 58,597 participants (58.7\% were women), with mean age ranging from $68.0 \pm 8.4$ to $87.3 \pm 3.7$. Thirty-six studies were cross-sectional and only one study 34 presented a retrospective longitudinal design.

Thirty-one studies 12,13,14,15,34,35,36,37,38,39,40,41,42,43,44,45,46,47,48,49,50,51,52,53,54,55,56,57,58,59,60 reported 12-month prevalence of falls, with estimates ranging between 6.5 and 46.9\%, and three studies 61,62,63 reported six-month prevalence, of falls, with estimates ranging between 21.5 and $53 \%$. The three remaining studies reported one-month prevalence 64 , three-month prevalence 65 , and lifetime-prevalence 66 of falls (Table 1). In this latter, the question on falls occurrence referred to the period after 60 years old 66. Descriptively, one-month prevalence was 11.4\% (95\%CI: 9.6-13.5) 64, three-month prevalence was 11.4\% (95\%CI: 9.4-13.6) 65, and lifetime-prevalence was 55.2\% (95\%CI: 50.7-59.7) 66. 
Figure 1

PRISMA flow of studies through the review.

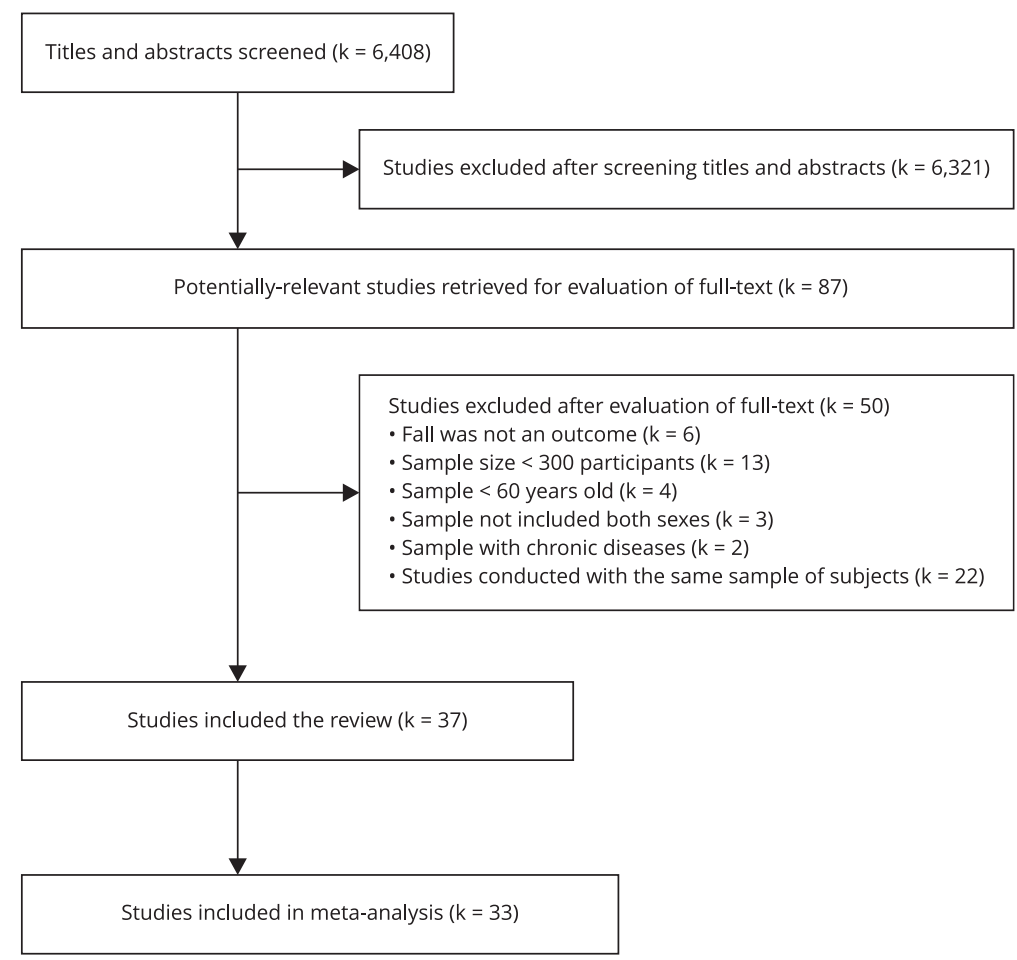

\section{Risk of bias analysis}

Assessment of methodological issues and bias risk in individual included studies provided low, unclear and high risk of bias ratings (Figure 2). Summarily, from 73 to $100 \%$ of studies were scored as low-risk on sample details, i.e. checklist items $1(\mathrm{k}=37), 2(\mathrm{k}=27)$, and $3(\mathrm{k}=37) ; 76$ and $95 \%$ were scored as low-risk on participants and sample coverage information, i.e. checklist items $4(\mathrm{k}=28)$ and $5(\mathrm{k}=35)$, respectively; 86 and $92 \%$ were scored as low-risk on measurement criteria and statistical analysis descriptions, i.e. checklist items $6(\mathrm{k}=32)$ and $8(\mathrm{k}=34)$, respectively; $62 \%$ scored as lowrisk and $30 \%$ scored as unclear on measurement reliability, i.e. checklist item 7 (low-risk, $\mathrm{k}=23$ and unclear, $\mathrm{k}=11$ ).

Two checklist questions had higher number of high-risk scores: $54 \%$ of studies were scored as high-risk on subgroup reporting, i.e. checklist items 9 and $10(\mathrm{k}=20)$. The main problems in these items were lack of data, absence of prevalence estimates by sex and age group and inconsistency between criteria for measurement of falls (e.g. considerable variation in age-range estimates across studies). Finally, the overall score for all included studies considering a mean of low-risk scores was 7.8 out of 10, ranging between 5 and 10 points (see supplementary data in Appendix 2 - http://cadernos. ensp.fiocruz.br/site/public_site/arquivo/supl-2-e00115718_2106.pdf). 


\section{Table 1}

Basic characteristics of the 37 included studies.

\begin{tabular}{|c|c|c|c|c|c|}
\hline \multirow[t]{2}{*}{ Study } & \multirow[t]{2}{*}{ Region } & \multirow{2}{*}{$\begin{array}{c}\text { Sample } \\
\text { n (F/M); age } \pm S D\end{array}$} & \multicolumn{3}{|c|}{ Prevalence (\%) } \\
\hline & & & Total & $\mathbf{F}$ & M \\
\hline \multicolumn{6}{|c|}{ One-month prevalence of falls } \\
\hline Cesar et al. 64 & $\mathrm{PA}, \mathrm{PI}$ & $1,013(376 / 637) ; 70.0 \pm 7.8$ years & 11.4 & NA & NA \\
\hline \multicolumn{6}{|c|}{ Three-month prevalence of falls } \\
\hline Benedetti et al. 65 & SC & 875 (438/437); $71.6 \pm 7.9$ years & 11.4 & 9.6 & 13.2 \\
\hline \multicolumn{6}{|c|}{ Six-month prevalence of falls } \\
\hline \multirow[t]{4}{*}{ Campos et al. 61} & MG & $2,052(1,226 / 826) ; 70.9 \pm 8.1$ years & 32.0 & 37.6 & 23.7 \\
\hline & & $60-69$ years & 29.3 & & \\
\hline & & 70-79 years & 33.0 & & \\
\hline & & $\geq 80$ years & 38.5 & & \\
\hline \multirow[t]{4}{*}{ Ribeiro et al. 62} & AM & $3,314(1,676 / 1,635) ; 72.4 \pm 7.8$ years & 21.5 & 26.3 & 22.7 \\
\hline & & 60-69 years & 21.4 & & \\
\hline & & $70-79$ years & 26.2 & & \\
\hline & & $\geq 80$ years & 29.0 & & \\
\hline Stamm et al. 63 & RS & $368(239 / 129) ; 71.9 \pm 7.7$ years & 53.0 & 37.8 & 15.2 \\
\hline \multicolumn{6}{|c|}{ 12-month prevalence of falls } \\
\hline \multirow[t]{4}{*}{ Antes et al. 35} & SC & $1,637(1,045 / 592) ; 70.7 \pm 8.0$ years & 19.0 & 21.5 & 14.3 \\
\hline & & 60-69 years & 13.5 & & \\
\hline & & 70-79 years & 17.6 & & \\
\hline & & $\geq 80$ years & 11.4 & & \\
\hline Aveiro et al. 36 & $\mathrm{SP}$ & 739 (434/305); $69.0 \pm 7.2$ years & 27.6 & 32.3 & 21.0 \\
\hline Berlezi et al. 37 & RS & $528(273 / 255) ; 72.3 \pm 9.4$ years & 19.9 & 25.3 & 14.1 \\
\hline \multirow[t]{4}{*}{ Brito et al. 38} & $\mathrm{BA}$ & $314(173 / 141) ; 74.2 \pm 9.7$ years & 25.8 & 32.3 & 17.7 \\
\hline & & $60-69$ years & 25.2 & & \\
\hline & & 70-79 years & 25.0 & & \\
\hline & & $\geq 80$ years & 27.3 & & \\
\hline \multirow[t]{4}{*}{ Carneiro et al. 39} & MG & $683(443 / 240) ; 70.9 \pm 8.0$ years & 28.4 & 32.5 & 20.8 \\
\hline & & $60-69$ years & 27.3 & & \\
\hline & & 70-79 years & 25.7 & & \\
\hline & & $\geq 80$ years & 37.7 & & \\
\hline Coimbra et al. 40 & SP & $2,209(1,332 / 877) ; 70.6 \pm 7.8$ years & 27.1 & NA & NA \\
\hline Confortin et al. 41 & SC & $1,656(1,058 / 598) ; 70.3 \pm 7.7$ years & 18.6 & NA & NA \\
\hline \multirow[t]{4}{*}{ Cruz et al. 12} & MG & $420(148 / 272) ; 69.7 \pm 6.9$ years & 32.1 & 37.1 & 23.0 \\
\hline & & $60-69$ years & 27.6 & & \\
\hline & & 70-79 years & 34.5 & & \\
\hline & & $\geq 80$ years & 44.4 & & \\
\hline \multirow[t]{3}{*}{ Dantas et al. 42} & PB & $401(271 / 130) ; 70.0 \pm 9.0$ years & 42.4 & 49.4 & 27.7 \\
\hline & & 60-69 years & 49.4 & & \\
\hline & & $\geq 70$ years & 38.7 & & \\
\hline \multirow[t]{4}{*}{ Gullich et al. 43} & SC & $552(301 / 251) ; N A$ & 28.3 & 30.9 & 25.1 \\
\hline & & $60-69$ years & 24.5 & & \\
\hline & & 70-79 years & 31.9 & & \\
\hline & & $\geq 80$ years & 38.6 & & \\
\hline Lebrão \& Laurenti 44 & $\mathrm{SP}$ & $2,143(1,255 / 888) ; 68.0 \pm 8.4$ years & 28.6 & 33.0 & 22.3 \\
\hline \multirow[t]{3}{*}{ Lima et al. 45} & $\mathrm{SP}$ & $432(263 / 169) ; 69.5 \pm 7.6$ years & 24.5 & 27.7 & 19.5 \\
\hline & & 60-69 years & 20.9 & & \\
\hline & & $\geq 70$ years & 30.5 & & \\
\hline Lima et al. 46 & RS & $418(239 / 181) ; 69 \pm 7.6$ years & 25.1 & NA & NA \\
\hline
\end{tabular}

(continues) 
Table 1 (continued)

\begin{tabular}{|c|c|c|c|c|c|}
\hline \multirow[t]{2}{*}{ Study } & \multirow[t]{2}{*}{ Region } & \multirow{2}{*}{$\begin{array}{c}\text { Sample } \\
\text { n (F/M); age } \pm \text { SD }\end{array}$} & \multicolumn{3}{|c|}{ Prevalence (\%) } \\
\hline & & & Total & $\mathbf{F}$ & M \\
\hline \multirow[t]{4}{*}{ Monego \& Barbosa 47} & SC & 477 (270/207); $74.2 \pm 8.4$ years & 24.3 & 31.2 & 15.7 \\
\hline & & 60-69 years & 21.0 & & \\
\hline & & 70-79 years & 22.3 & & \\
\hline & & $\geq 80$ years & 41.8 & & \\
\hline Moreira et al. 34 * & $\mathrm{RJ}$ & $490(374 / 116) ; 79.0 \pm$ NA years & 27.9 & 31.0 & 18.1 \\
\hline \multirow[t]{4}{*}{ Motta et al. 48} & RJ & $1,064(606 / 458) ; 71.4 \pm 8.0$ years & 30.3 & 36.1 & 22.7 \\
\hline & & 60-69 years & 28.3 & & \\
\hline & & 70-79 years & 31.5 & & \\
\hline & & $\geq 80$ years & 34.0 & & \\
\hline \multirow[t]{3}{*}{ Nascimento \& Tavares 49} & MG & 729 (487/242); NA & 28.3 & 33.1 & 18.6 \\
\hline & & 60-80 years & 26.4 & & \\
\hline & & $\geq 80$ years & 35.7 & & \\
\hline \multirow[t]{4}{*}{ Nunes et al. 50} & RS & $1,591(1,000 / 591) ; 70.6 \pm 8.1$ years & 28.0 & 33.3 & 19.1 \\
\hline & & 60-69 years & 24.0 & & \\
\hline & & 70-74 years & 28.9 & & \\
\hline & & $\geq 75$ years & 33.8 & & \\
\hline \multirow[t]{4}{*}{ Nunes et al. 51} & GO & $388(227 / 161) ; 69.7 \pm 7.5$ years & 38.7 & 42.7 & 32.9 \\
\hline & & 60-69 years & 34.1 & & \\
\hline & & 70-79 years & 40.3 & & \\
\hline & & $\geq 80$ years & 56.5 & & \\
\hline \multirow[t]{4}{*}{ Pereira et al. 52} & RS & $6,751(3,494 / 3,257) ; 70.0 \pm 7.3$ years & 10.7 & 13.4 & 10.4 \\
\hline & & 60-69 years & 6.4 & & \\
\hline & & 70-79 years & 13.4 & & \\
\hline & & $\geq 80$ years & 20.9 & & \\
\hline \multirow[t]{3}{*}{ Pereira et al. 53} & PR & $350(212 / 138) ; 87.3 \pm 3.7$ years & 46.9 & NA & NA \\
\hline & & 80-89 years & 49.4 & & \\
\hline & & $\geq 90$ years & 17.9 & & \\
\hline Perracini \& Ramos 54 & SP & $1,415(917 / 498) ; 70.0 \pm$ NA years & 30.9 & NA & NA \\
\hline \multirow[t]{4}{*}{ Pimentel et al. 55} & $\mathrm{AL}, \mathrm{AM}, \mathrm{BA}, \mathrm{CE}, \mathrm{ES}, \mathrm{DF}$, & $4,174(2,362 / 1,812) ; 70.2 \pm$ NA years & 25.1 & 30.2 & 18.4 \\
\hline & GO, MA, MG, MS, MT, & 60-64 years & 23.6 & & \\
\hline & $\mathrm{PA}, \mathrm{PB}, \mathrm{PE}, \mathrm{Pl}, \mathrm{PR}, \mathrm{RJ}$ & $65-74$ years & 22.1 & & \\
\hline & RN, RS, SC, SE, SP & $\geq 75$ years & 31.4 & & \\
\hline Rodrigues et al. 56 & $\mathrm{SP}$ & $1,520(904 / 616) ; 69.9 \pm 7.8$ years & 6.5 & 8.7 & 3.4 \\
\hline \multirow[t]{4}{*}{ Sampaio et al. 57} & RJ, MG, PR, SC, SP & $578(456 / 122) ; 70.0 \pm 6.6$ years & 22.5 & 24.8 & 13.9 \\
\hline & & 60-69 years & 20.3 & & \\
\hline & & 70-79 years & 24.4 & & \\
\hline & & $\geq 80$ years & 33.3 & & \\
\hline \multirow[t]{4}{*}{ Sandoval et al. 58} & GO & 938 (570/348); $71.5 \pm 8.4$ years & 34.0 & 37.9 & 29.6 \\
\hline & & 60-69 years & 29.2 & & \\
\hline & & 70-79 years & 37.1 & & \\
\hline & & $\geq 80$ years & 45.3 & & \\
\hline Silva et al. 59 & $\begin{array}{c}C E, M G, P A, P E, P I, R N, \\
R S, S P\end{array}$ & $5,532(3,629 / 1,903) ; 73.1 \pm 6.2$ years & 29.2 & NA & NA \\
\hline \multirow[t]{4}{*}{ Siqueira et al. 15} & $A C, A L, B A, C E, E S, G O$, & $6,616(3,903 / 2,713) ; 70.9 \pm 7.9$ years & 27.6 & 32.1 & 21.2 \\
\hline & $\mathrm{MA}, \mathrm{MG}, \mathrm{MS}, \mathrm{PA}, \mathrm{PB}$ & 60-69 years & 24.4 & & \\
\hline & $P E, P I, P R, R J, R N, R O$ & 70-79 years & 27.9 & & \\
\hline & RS, SC, SE, SP, TO & $\geq 80$ years & 37.1 & & \\
\hline \multirow[t]{5}{*}{ Siqueira et al. 14} & $A L, P B, P E, P I, R N$ & $4,003(2,450 / 1,553) ; 73.9 \pm 7.0$ years & 34.8 & 40.1 & 26.5 \\
\hline & $\mathrm{RS}, \mathrm{SC}$ & $65-70$ years & 31.8 & & \\
\hline & & $71-75$ years & 33.2 & & \\
\hline & & $76-80$ years & 36.8 & & \\
\hline & & $\geq 80$ years & 42.0 & & \\
\hline
\end{tabular}

(continues) 
Table 1 (continued)

\begin{tabular}{|c|c|c|c|c|c|}
\hline \multirow[t]{2}{*}{ Study } & \multirow[t]{2}{*}{ Region } & \multirow{2}{*}{$\begin{array}{c}\text { Sample } \\
\text { n (F/M); age } \pm \text { SD }\end{array}$} & \multicolumn{3}{|c|}{ Prevalence (\%) } \\
\hline & & & Total & $\mathbf{F}$ & M \\
\hline Vieira et al. 60 & RS & $1,451(914 / 537) ; 70.7 \pm 8.2$ years & 28.1 & NA & NA \\
\hline Zazzetta et al. 13 & SP & $304(173 / 131) ; 70.1 \pm 7.6$ years & 16.1 & 19.0 & 13.0 \\
\hline \multicolumn{6}{|c|}{ Lifetime-prevalence of falls } \\
\hline Chehuen Neto et al. 66 & MG & $472(276 / 285) ; 70.6 \pm$ NA years & 55.1 & 55.1 & 55.1 \\
\hline
\end{tabular}

F: female; M: male; NA: not available; SD: standard deviation.

Note: Brazilian states: AC: Acre; AL: Alagoas; AM: Amazonas; BA: Bahia; CE: Ceará; DF: Federal District; ES: Espírito Santo; GO: Goiás; MA: Maranhão; MG: Minas Gerais; MS: Mato Grosso do Sul; MT: Mato Grosso; PA: Pará; PB: Paraíba; PE: Pernambuco; PI: Piauí; PR: Paraná; RJ: Rio de Janeiro; RN: Rio Grande do Norte; RO: Rondônia; RS: Rio Grande do Sul; SC: Santa Catarina; SE: Sergipe; SP: São Paulo; TO: Tocantins.

* Study with a retrospective longitudinal design.

\section{Figure 2}

Risk of bias summary of the included studies $(\mathrm{k}=37)$

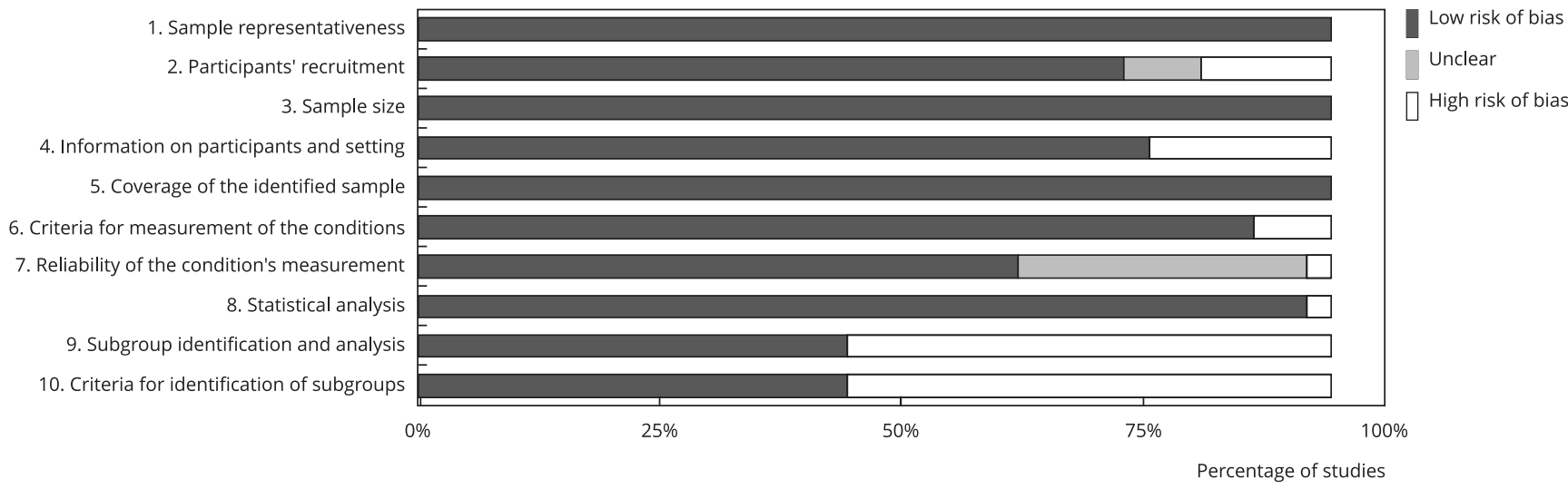

\section{Pooled analysis}

Meta-analysis was performed pooling data from the 30 studies reporting at least one event of fall in the preceding 12 months and from the three studies reporting at least one event of fall in the preceding six months. The estimates revealed that at 12 -month period, prevalence of falls was $27 \%$ (95\% CI: 24.3-30.0) (Figure 3). No important asymmetry that could indicate publication bias was observed by inspecting funnel plot for the 12-month estimates (see supplementary data in Appendix 3 - http:// cadernos.ensp.fiocruz.br/site/public_site/arquivo/supl-3-e00115718_8221.pdf). According to the GRADE system, these pooled studies provide high-quality evidence that the 12 -month prevalence of falls in older Brazilians is $27 \%$.

At six-month period, prevalence of falls was 34.3\% (95\%CI: 21.9-49.3) (see supplementary data in Appendix 4 - http://cadernos.ensp.fiocruz.br/site/public_site/arquivo/supl-4-e00115718_2678. pdf). According to the GRADE system, these pooled studies provide low-quality evidence that the six-month prevalence of falls in older Brazilians is 34.3\%. The evidence was downgraded from highto low-quality because of inconsistency between studies ( $\left.\mathrm{I}^{2}=40 \%\right)$ and impossibility to evaluate publication bias. 
Figure 3

Meta-analysis including studies reporting 12-month prevalence of falls $(k=31)$.

\begin{tabular}{|c|c|c|c|c|}
\hline Study & Estimate $\%(95 \% \mathrm{Cl})$ & Random-effects & Event/Total & Weight $\%$ \\
\hline Antes et al. 35 & $19.0(17.1-20.9)$ & $\square$ & $312 / 1,637$ & 3.28 \\
\hline Aveiro et al. ${ }^{36}$ & $27.6(24.5-30.9)$ & $=$ & $204 / 739$ & 3.23 \\
\hline Berlezi et al. ${ }^{37}$ & $19.9(16.7-23.5)$ & $=$ & $105 / 528$ & 3.15 \\
\hline Brito et al. ${ }^{38}$ & $25.8(21.3-30.9)$ & $=$ & $81 / 314$ & 3.07 \\
\hline Carneiro et al. ${ }^{39}$ & $28.4(25.1-31.9)$ & $=$ & $194 / 683$ & 3.23 \\
\hline Coimbra et al. ${ }^{40}$ & $27.1(25.3-29.0)$ & $\square$ & $599 / 2,209$ & 3.31 \\
\hline Confortin et al. ${ }^{41}$ & $18.6(16.8-20.5)$ & - & $308 / 1,656$ & 3.28 \\
\hline Cruz et al. 12 & $32.1(27.8-36.8)$ & - & $135 / 420$ & 3.16 \\
\hline Dantas et al. ${ }^{42}$ & $42.4(37.6-47.3)$ & -2 & $170 / 401$ & 3.18 \\
\hline Gullich et al. ${ }^{43}$ & $28.3(24.7-32.2)$ & $\Rightarrow$ & $156 / 552$ & 3.20 \\
\hline Lebrão \& Laurenti 44 & $28.6(26.7-30.6)$ & $\square$ & $613 / 2,143$ & 3.31 \\
\hline Lima et al. ${ }^{45}$ & $24.5(20.7-28.8)$ & $=$ & $106 / 432$ & 3.14 \\
\hline Lima et al. ${ }^{46}$ & $25.1(21.2-29.5)$ & 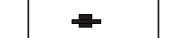 & $105 / 418$ & 3.13 \\
\hline Monego \& Barbosa 47 & $24.3(20.7-28.4)$ & $=$ & $16 / 1,477$ & 3.16 \\
\hline Moreira et al. ${ }^{34}$ & $27.9(24.2-32.1)$ & $=$ & $137 / 490$ & 3.18 \\
\hline Motta et al. ${ }^{48}$ & $30.3(27.6-33.1)$ & 들 & $322 / 1,064$ & 3.28 \\
\hline Nascimento \& Tavares ${ }^{49}$ & $28.3(25.1-31.6)$ & $=$ & $206 / 729$ & 3.23 \\
\hline Nunes at al. ${ }^{50}$ & $28.0(25.9-30.3)$ & 를 & $446 / 1,591$ & 3.30 \\
\hline Nunes et al. ${ }^{51}$ & $38.7(33.9-43.6)$ & 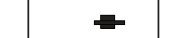 & $150 / 388$ & 3.17 \\
\hline Pereira et al. ${ }^{52}$ & $10.7(9.9-11.4)$ & $\mathbf{\square}$ & $719 / 6,751$ & 3.33 \\
\hline Pereira et al. ${ }^{53}$ & $46.9(41.7-52.1)$ & $\Rightarrow$ & $164 / 350$ & 3.16 \\
\hline Perracini \& Ramos 54 & $30.9(28.5-33.3)$ & 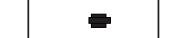 & $437 / 1,415$ & 3.30 \\
\hline Pimentel et al. ${ }^{55}$ & $25.1(23.8-26.4)$ & $\mathbf{\square}$ & $1,048 / 4,174$ & 3.33 \\
\hline Sampaio et al. ${ }^{57}$ & $22.5(19.3-26.1)$ & $=$ & $130 / 578$ & 3.18 \\
\hline Sandoval et al. ${ }^{58}$ & $34.0(31.0-37.1)$ & $=$ & $319 / 938$ & 3.27 \\
\hline Silva et al. 59 & $29.2(28.0-30.4)$ & - & $1,615 / 5,532$ & 3.34 \\
\hline Siqueira et al. ${ }^{15}$ & $27.6(26.5-28.7)$ & $\square$ & $1,826 / 6,616$ & 3.34 \\
\hline Siqueira et al. ${ }^{14}$ & $34.8(33.4-36.3)$ & a & $1,394 / 4,003$ & 3.34 \\
\hline Vieira et al. ${ }^{60}$ & $28.1(25.9-30.5)$ & $=$ & $408 / 1,451$ & 3.29 \\
\hline Zazzetta et al. ${ }^{13}$ & $16.1(12.4-20.7)$ & & $49 / 304$ & 2.96 \\
\hline Pooled effect & $27.0(24.3-30.0)$ & $<$ & $12,666 / 50,503$ & \\
\hline \multicolumn{2}{|c|}{$\begin{array}{l}\operatorname{Tau}^{2}=0.00 ; Q=27.26 ; d f=29(p=0.56) ; I^{2}=0.00 \% \\
\text { Test for pooled effect } z=-13.59(p<0.001)\end{array}$} & $\begin{array}{l}0.00 \quad 60.00 \\
\text { Prevalence } \%\end{array}$ & & \\
\hline
\end{tabular}

\section{Exploratory analysis}

Subgroup analysis evidenced that fall events at 12-month period were significantly more common in female than male $(30.8 \%$ vs $19.8 \%, P R=1.57)$, in those with $\geq 80$ years than those with $60-69$ years $(35.7 \%$ vs $23.8 \%$, PR $=1.46)$, and in participants from the Central region than participants from the South region $(33.7 \%$ vs $24 \%$, PR $=1.36)$ of Brazil (Table 2 ; and supplementary data in Appendix 5 - http://cadernos.ensp.fiocruz.br/site/public_site/arquivo/supl-5-e00115718_1544.pdf, Appendix 6 - http://cadernos.ensp.fiocruz.br/site/public_site/arquivo/supl-6-e00115718_5129.pdf, and Appendix 7 - http://cadernos.ensp.fiocruz.br/site/public_site/arquivo/supl-7-e00115718_6695. pdf). Meta-regression analysis found no association between risk of bias scores and heterogeneity regarding 12-month prevalence estimates ( $\beta=0.055,95 \% \mathrm{CI}:-0.084-0.193, \mathrm{p}=0.42, \mathrm{R}^{2}=0.00$ ). 
Table 2

Results from meta-analyses of subgroups including studies reporting 12-month prevalence of falls $(k=30)$.

\begin{tabular}{|c|c|c|c|c|}
\hline Subgroup & $\begin{array}{l}\text { Estimate * } \\
\%(95 \% \mathrm{Cl})\end{array}$ & $1^{2}$ & PR $(95 \% \mathrm{Cl})$ & p-value \\
\hline \multicolumn{5}{|l|}{ Sex } \\
\hline Male $(k=23)$ & $19.8(17.1-22.8)$ & $0 \%$ & 1.00 & \\
\hline Female $(k=23)$ & $30.8(27.2-34.6)$ & $0 \%$ & $1.57(1.32-1.86)$ & $<0.001$ \\
\hline \multicolumn{5}{|l|}{ Age (years) } \\
\hline $60-69(k=15)$ & $23.8(18.9-29.5)$ & $2 \%$ & 1.00 & \\
\hline $70-79(k=12)$ & $26.6(20.6-33.5)$ & $0 \%$ & $1.09(0.86-1.38)$ & 0.494 \\
\hline$\geq 80(k=15)$ & $35.7(31.1-42.8)$ & $0 \%$ & $1.46(1.15-1.84)$ & 0.002 \\
\hline \multicolumn{5}{|l|}{ Region } \\
\hline North ** $(k=1)$ & $18.6(16.3-21.1) * * *$ & - & - & \\
\hline South $(k=11)$ & $24.0(20.1-28.3)$ & $22 \%$ & 1.00 & \\
\hline Southeast $(k=13)$ & $28.0(24.0-32.3)$ & $27 \%$ & $1.06(0.83-1.35)$ & 0.628 \\
\hline Northeast (k = 4) & $31.4(23.9-40.0)$ & $0 \%$ & $1.27(0.96-1.68)$ & 0.101 \\
\hline Central $(k=4)$ & $33.7(25.9-42.5)$ & $0 \%$ & $1.36(1.10-1.69)$ & 0.006 \\
\hline
\end{tabular}

95Cl: 95\% confidence interval; 12: statistics for measuring heterogeneity within and among studies; PR: prevalence ratio (based on Poisson regression with robust estimation of variance).

* Estimates are from random-effects meta-analysis;

* North region was not accounted for PR estimates because only one study was available for meta-analysis;

** Estimate is only descriptive.

\section{Discussion}

The present study aimed at investigating the prevalence of falls and associated factors in communitydwelling older people living in Brazil. Pooled estimates evidenced a 12-month prevalence of $27 \%$, with events occurring more frequently in women, in those with advanced age, and in participants from the Central region of Brazil. No association was found between risk of bias ratings and the 12-month prevalence estimates.

From the authors' knowledge, this is the first meta-analysis estimating prevalence of falls in older Brazilians. The review findings revealed values very close to those observed in other countries with socioeconomic levels similar to Brazil. Twelve-month prevalence was $26.8 \%$ in Mexico $67,28.5 \%$ in Turkey 68 , and $26.4 \%$ in South Africa 69 . The results also agreed with those of international studies. The prevalence was 28.4\% in England 70, 24.9\% in France 71, 24.4\% in Australia 72 and 29.1\% in Spain 73. Comparing these results with other studies, the findings also demonstrated that falling is one of the most important health outcomes that affect older Brazilians during their life, exceeding other adverse conditions such as sarcopenia (17\%) 74 , frailty (17.9\%) 75 and osteoporosis (14.8\%) 76 .

Subgroup analysis suggested that being a woman may be a risk factor for falling, corroborating literature reports 77,78 . The results revealed that women are $60 \%$ more likely to fall than men. Increased longevity is associated with a higher prevalence of chronic conditions that are strongly associated with increased risk of functional disability and mortality, especially in women 79 .

Greater longevity may culminate in more severe repercussions for motor and sensory functions such as gait pattern, postural control and quadriceps, plantar flexor and dorsiflexor muscle strength, which are responsible for the ankle strategy commonly used by older adults to maintain dynamic and semi-static postural balance 80 . Moreover, falls exhibit multifactorial etiology, including a range of changes in physiological factors (e.g. increased fat mass, bone loss and uncontrolled blood glucose) 57,81,82. For example, estrogen hormones, which exert a protective effect against Alzheimer's disease, decline progressively during the climacteric period, thereby increasing the risk of falling 83 . 
The analysis for age group demonstrated that advanced age is associated with falls prevalence, as already shown in previous studies 81,84 . In individuals aged 80 years or older, the odds of falling was greater than in those aged between 60 and 69 years 12,84. Physiological changes such as slow pupillary reactions, neuronal loss and vestibular dysfunction, which lead to slowness in neural responses, decrease in bone mineral density and reduction of type II muscle fibers are also highlighted 1.

A national health survey conducted in Brazil in 2013 showed that chronic diseases such as diabetes mellitus increase with advanced age 85 , which can lead to complications such as neuropathy and retinopathy 86 . A recent meta-analysis revealed that diabetes mellitus is a strong predictor for falls $(\mathrm{RR}=1.64,95 \% \mathrm{CI}: 1.27-2.11) 82$. Other chronic diseases, such as osteoporosis and osteoarthritis, also make the older person more vulnerable to falls 76,80 . Aging is also associated to sarcopenia, a geriatric syndrome characterized by loss of muscle mass and function that becomes more intense over time and is independently associated with higher rates of falls (HR $=3.23,95 \% \mathrm{CI}: 1.25-8.29) 87$.

Twelve-month was the most widely used period in literature when investigating falls prevalence. Studies have shown that the occurrence of an event with adverse repercussions is vivid in the memory of participants $15,33,68$. On the other hand, the prevalence of falls may be underestimated due to falls without repercussions 88 . Therefore, standardizing prevalence measurements and additional care during data collection are strongly recommended in research and clinical practice to obtain more conservative estimates.

With respect to geographic region, it was expected that less developed locations would exhibit higher prevalence estimates 89 . However, the findings diverged. The North region had lower prevalence (18.6\%) than the national estimate on 12 -month period. One of the possible explanations for this result is that only one study represented the North region and sociodemographic and clinical factors of the study sample (e.g. age, sex, and health status) may have influenced the estimate. Another two included studies estimated the prevalence of falls in the North region at a one-month (11.4\%) 64 and at a six-month period (21.5\%) 62. Future surveys in this region covering more number of states and using standardized measurements may provide more accurate results on falls prevalence.

Potential limitations of the review need to be discussed. First, most of the included studies were from more socially and economically developed areas, such as South and Southeast regions of Brazil, which accounted for around $80 \%$ of the estimates pooled in the meta-analysis. Future investigations in less developed regions are needed to obtain more information about the epidemiology of falls in older individuals residing in vulnerable locations. Second, low-quality evidence reinforces that the six-month pooled estimate might not reflect an accurate result, especially, due to small number of studies included in the meta-analysis $(\mathrm{k}=3)$, important heterogeneity among studies ( $\left.\mathrm{I}^{2}=40 \%\right)$, and impossibility for assessing publication bias. Third, a range of clinical factors such as functional status, use of medications and presence of comorbidities may also have impacted the prevalence estimates 90 . Unfortunately, methodological inconsistencies among the included studies did not allow a secondary analysis encompassing these information. For example, one study addressed functional performance through the Lawton and Brody Scale 12, others using the questionnaire Old Americans Resources and Services 41 or Time UP and Go Test 36 , and others did not evaluate functional outcomes 15,40,42,43.

Finally, strengths should also be mentioned. First, the descriptive findings of the review raise important questions about methodological and sociodemographic aspects involved in the epidemiology of falls in older Brazilians, which can direct new research with insights to address literature gaps (e.g. lack of standardized prevalence measurements and scarcity of epidemiological data by geographic region). Second, the 12-month prevalence of falls was obtained by pooling data from well-designed cross-sectional studies including representative samples from several communities in Brazil. This provides adequate statistical power and generability for 12-month estimate and thus it fulfills the main objective of the present review. Third, exploratory analysis enhances the review's scope by accounting for well-known factors related to falls occurrence in the older population (e.g. sex and age). 


\section{Conclusion}

High-quality evidence indicates that one in four community-dwelling older people from Brazil experiences at least one episode of fall in the preceding 12 months. In addition, despite of ecological limitations, additional findings support the attention that must be given to women and those aged 80 years and older as the Brazilian population ages. Clinicians, researchers, managers, policymakers and patients should collaborate for developing preventive, educational and promotional health programs to reduce the prevalence of falls as well as the associated adverse outcomes.

\section{Contributors}

J. Elias Filho participated in research in databases, article selections, data extraction, statistical analysis, and writing. W. P. Borel participated in research in databases, article selections, and data extraction. J. B. M. Diz, A. W. C. Barbosa, and R. R. Britto contributed to all the stages and participated in the interpretation of the results. D. C. Felício conceived the study, participated in all the stages and contributed to the writing.

\section{Additional informations}

ORCID: José Elias Filho (0000-0002-4251-0290); Wyngrid Porfirio Borel (0000-0002-1182-9856); Juliano Bergamaschine Mata Diz (0000-00022849-2081); Alexandre Wesley Carvalho Barbosa (0000-0001-7862-1737); Raquel Rodrigues Britto (0000-0002-9533-3654); Diogo Carvalho Felício (0000-0001-5138-1884).

\section{Acknowledgments}

This study was financed in part by the Brazilian Graduate Studies Coordinating Board (Capes; Finance Code 001).

\section{References}

1. Silva Gama ZA, Gomez Conesa A, Sobral Ferreira M. Epidemiology of falls in the elderly in Spain: a systematic review, 2007. Rev Esp Salud Pública 2008; 82:43-55.

2. Hartholt KA, van Beeck EF, Polinder S, van der Velde N, van Lieshout EM, Panneman MJ, et al. Societal consequences of falls in the older population: injuries, healthcare costs, and long-term reduced quality of life. J Trauma 2011; 71:748-53.

3. Caberlon IC, Bós ÂJG. Diferenças sazonais de quedas e fraturas em idosos gaúchos. Ciênc Saúde Colet 2015; 20:3743-52.

4. Ferretti F, Lunardi D, Bruschi L. Causes and consequences of fall among elderly people at home. Fisioter Mov 2013; 26:753-62.

5. Barros IFO, Pereira MB, Weiller TH, Anversa ETR. Internações hospitalares por quedas em idosos brasileiros e os custos correspondentes no âmbito do Sistema Único de Saúde. Rev Kairós 2015; 18:63-80.

6. Andrade IR, Souza EA, Luz LA, Pinto Junior E. Características e gastos com hospitalizações por quedas em idosos na Bahia. J Health Sci Inst 2017; 35:28-31.

7. Brasil. Lei no 8.842 , de 4 de janeiro de 1994. Dispõe sobre a Política Nacional do Idoso, cria o Conselho Nacional do Idoso e dá outras providências. Diário Oficial da União 1994; 5 jan.

8. Ministério da Saúde. Portaria GM no 1.395, de 9 de dezembro de 1999, que aprova a Política Nacional de Saúde do Idoso e dá outras providências. Diário Oficial da União 1999; 13 dec.

9. Brasil. Lei no 10.741, de 1o de outubro de 2003. Dispõe sobre o Estatuto do Idoso e dá outras providências. Diário Oficial da União 2003; 3 oct.

10. Brasil. Constituição da República Federativa do Brasil. Brasília: Senado Federal; 1988. 
11. Santos RKM, Maciel ACC, Britto HMJ, Lima JCC, Souza TO. Prevalência e fatores associados ao risco de quedas em idosos adscritos a uma Unidade Básica de Saúde do município de Natal, RN, Brasil. Ciênc Saúde Colet 2015; 20:3753-62.

12. Cruz DT, Ribeiro LC, Vieira MT, Teixeira MTB, Bastos RR, Leite ICG. Prevalence of falls and associated factors in elderly individuals. Rev Saúde Pública 2012; 46:138-46.

13. Zazzetta MS, Gomes GA, Orlandi FS, Gratao AC, Vasilceac FA, Gramani-Say K, et al. Identifying frailty levels and associated factors in a population living in the context of poverty and social vulnerability. J Frailty Aging 2017; 6:2932 .

14. Siqueira FV, Facchini LA, Piccini RX, Tomasi E, Thumé E, Silveira DS, et al. Prevalência de quedas em idosos e fatores associados. Rev Saúde Pública 2007; 41:749-56.

15. Siqueira FV, Facchini LA, Silveira DS, Piccini RX, Tomasi E, Thume E, et al. Prevalence of falls in elderly in Brazil: a countrywide analysis. Cad Saúde Pública 2011; 27:1819-26.

16. Munn Z, Moola S, Lisy K, Riitano D. The Joanna Briggs Institute Reviewers' Manual 2014. The systematic review of prevalence and incidence data. Adelaide: The Joanna Briggs Institute; 2014

17. Munn Z, Moola S, Riitano D, Lisy K. The development of a critical appraisal tool for use in systematic reviews addressing questions of prevalence. Int J Health Policy Manag 2014; 3:123-8.

18. Stroup DF, Berlin JA, Morton SC, Olkin I, Williamson GD, Rennie D, et al. Meta-analysis of observational studies in epidemiology: a proposal for reporting. Meta-analysis Of Observational Studies in Epidemiology (MOOSE) group. JAMA 2000; 283:2008-12.

19. Higgins JP, Green S, editors. Cochrane handbook for systematic reviews of interventions, Version 5.1.0 [update March 2011]. http:// handbook.cochrane.org/ (accessed on 12/Jan/ 2019).

20. Moher D, Liberati A, Tetzlaff J, Altman DG. Preferred reporting items for systematic reviews and meta-analyses: the PRISMA statement. J Clin Epidemiol 2009; 62:1006-12.

21. Arya R, Antonisamy B, Kumar S. Sample size estimation in prevalence studies. Indian J Pediatr 2012; 79:1482-8.

22. Loney PL, Chambers LW, Bennett KJ, Roberts JG, Stratford PW. Critical appraisal of the health research literature: prevalence or incidence of a health problem. Chronic Dis Can 1998; 19:170-6.

23. Chinnakali P, Yadav K, Singh AK. Importance of reporting age-adjusted prevalence in epidemiological studies. N Am J Med Sci 2012; 4:107-8.

24. Kasten AP, Rosa BN, Schmit EFD, Noll M, Candotti CT. Prevalence of postural deviations in the spine in schoolchildren: a systematic review with meta-analysis. J Hum Growth Dev 2017; 27:99-108.
25. Gorter TM, Hoendermis ES, van Veldhuisen DJ, Voors AA, Lam CS, Geelhoed B, et al. Right ventricular dysfunction in heart failure with preserved ejection fraction: a systematic review and meta-analysis. Eur J Heart Fail 2016; 18:1472-87.

26. Leopoldino AA, Diz JB, Martins VT, Henschke N, Pereira LS, Dias RC, et al. Prevalence of low back pain in older Brazilians: a systematic review with meta-analysis. Rev Bras Reumatol Engl Ed 2016; 56:258-69.

27. Simmonds M. Quantifying the risk of error when interpreting funnel plots. Syst Rev 2015; $4: 24$.

28. Coutinho LM, Scazufca M, Menezes PR. Methods for estimating prevalence ratios in cross-sectional studies. Rev Saúde Pública 2008; 42:992-8

29. Jackson D, White IR, Thompson SG. Extending DerSimonian and Laird's methodology to perform multivariate random effects metaanalyses. Stat Med 2010; 29:1282-97.

30. Viechtbauer W. Bias and efficiency of metaanalytic variance estimators in the randomeffects model. J Educ Behav Stat 2005; 30:26193.

31. Knapp G, Hartung J. Improved tests for a random effects meta-regression with a single covariate. Stat Med 2003; 22:2693-710.

32. Balshem H, Helfand M, Schunemann HJ, Oxman AD, Kunz R, Brozek J, et al. GRADE guidelines: 3 . Rating the quality of evidence. J Clin Epidemiol 2011; 64:401-6.

33. Mackenzie L, Byles J, D’Este C. Validation of self-reported fall events in intervention studies. Clin Rehabil 2006; 20:331-9.

34. Moreira MD, Costa AR, Caldas CP. The association between nursing diagnoses and the occurrence of falls observed among eldery individuals assisted in an outpatient facility. Rev Latinoam Enferm 2007; 15:311-7.

35. Antes DL, d'Orsi E, Benedetti TRB. Circumstances and consequences of falls among the older adults in Florianopolis. EpiFloripa Aging 2009. Rev Bras Epidemiol 2013; 16:469-81.

36. Aveiro MC, Driusso P, Barham EJ, Pavarini SCI, Oishi J. Mobilidade e risco de quedas de população idosa da comunidade de São Carlos. Ciênc Saúde Colet 2012; 17:2481-8.

37. Berlezi EM, Farias AM, Dallazen F, Oliveira KR, Pillatt AP, Fortes CK. Analysis of the functional capacity of elderly residents of communities with a rapid population aging rate. Rev Bras Geriatr Gerontol 2016; 19:643-52.

38. Brito TA, Coqueiro RS, Fernandes MH, Jesus CS. Determinants of falls in communitydwelling elderly: hierarchical analysis. Public Health Nurs 2014; 31:290-7.

39. Carneiro JA, Ramos GCF, Barbosa ATF, Vieira EDS, Silva JSR, Caldeira AP. Falls among the non-institutionalized elderly in northern Minas Gerais, Brazil: prevalence and associated factors. Rev Bras Geriatr Gerontol 2016; 19:613-25. 
40. Coimbra AM, Ricci NA, Coimbra IB, Costallat LT. Falls in the elderly of the Family Health Program. Arch Gerontol Geriatr 2010; 51:31722.

41. Confortin SC, Giehl MWC, Antes DL, Schneider IJC, d'Orsi E. Positive self-rated health in the elderly: a population-based study in the South of Brazil. Cad Saúde Pública 2015; 31:1049-60.

42. Dantas EL, Brito GEG, Lobato IAF. Prevalência de quedas em idosos adscritos à estratégia de saúde da família do município de João Pessoa, Paraíba. Rev APS 2012; 15:67-75.

43. Gullich I, Duro SMS, Cesar JA. Depression among the elderly: a population-based study in Southern Brazil. Rev Bras Epidemiol 2016; 19:691-701.

44. Lebrão ML, Laurenti R. Saúde, bem-estar e envelhecimento: o estudo SABE no Município de São Paulo. Rev Bras Epidemiol 2005; 8:127-41.

45. Lima AP, Lini EV, Tomicki C, Dellani M, Portella $\mathrm{M}$, Doring $\mathrm{M}$. Fatores associados à atividade física em idosos de Estação, Rio Grande do Sul: estudo de base populacional. Rev Bras Ativ Fis Saúde 2016; 20:618-25.

46. Lima MCP, Simão MO, Oliveira JB, Cavariani MB, Tucci AM, Kerr-Correa F. Alcohol use and falls among the elderly in Metropolitan São Paulo, Brazil. Cad Saúde Pública 2009; 25:2603-11.

47. Monego EA, Barbosa AR. Factors associated with daily sitting time in a rural communitydwelling of older adults from southern Brazil. Rev Bras Ativ Fís Saúde 2014; 19:371-81.

48. Motta LB, Aguiar AC, Coutinho ESF, Huf G. Prevalência e fatores associados a quedas em idosos em um município do Rio de Janeiro. Rev Bras Geriatr Gerontol 2010; 13:83-91.

49. Nascimento JS, Tavares DMS. Prevalence and factors associated with falls in the elderly. Texto Contexto Enferm 2016; 25:e0360015.

50. Nunes BP, Saes MO, Siqueira FV, Tomasi E, Silva SM, Silveira DS, et al. Falls and self-assessment of eyesight among elderly people: a population-based study in a south Brazilian municipality. Arch Gerontol Geriatr 2014; 59:131-5.

51. Nunes DP, Nakatani AYK, Silveira EA, Bachion MM, Souza MR. Capacidade funcional, condições socioeconômicas e de saúde de idosos atendidos por equipes de Saúde da Família de Goiânia (GO, Brasil). Ciênc Saúde Colet 2010; 15:2887-98.

52. Pereira GN, Morsch P, Lopes DGC, Trevisan MD, Ribeiro A, Navarro JHN, et al. Fatores socioambientais associados à ocorrência de quedas em idosos. Ciênc Saúde Colet 2013; 18:3507-14.

53. Pereira SG, Santos CB, Doring M, Portella MR. Prevalence of household falls in longlived adults and association with extrinsic factors. Rev Latinoam Enferm 2017; 25:e2900.

54. Perracini MR, Ramos LR. Fatores associados a quedas em uma coorte de idosos residentes na comunidade. Rev Saúde Pública 2002; 36:70916.
55. Pimentel WRT, Pagotto V, Stopa SR, Hoffmann MCCL, Andrade FB, Souza Junior PRB, et al. Falls among Brazilian older adults living in urban areas: ELSI-Brazil. Rev Saúde Pública 2018; 52 Suppl 2:12s.

56. Rodrigues IG, Fraga GP, Barros MB. Falls among the elderly: risk factors in a population-based study. Rev Bras Epidemiol 2014; 17:705-18

57. Sampaio RAC, Sampaio PYS, Castano LAA, Barbieri JF, Coelho HJJ, Arai H, et al. Cutoff values for appendicular skeletal muscle mass and strength in relation to fear of falling among Brazilian older adults: cross-sectional study. São Paulo Med J 2017; 135:434-43.

58. Sandoval RA, Menezes R, Pagotto V, Nakatani AYK, Bachion M. Quedas de idosos não institucionalizados e fatores associados: estudo de base populacional em uma metrópole da região Centro-Oeste no Brasil. Rev Cient Esc Estadual Saúde Pública de Goiás Cândido Santiago 2015; 1:91-107.

59. Silva SLA, Neri AL, Ferrioli E, Lourenço RA, Dias RC. Fenótipo de fragilidade: influência de cada item na determinação da fragilidade em idosos comunitários - Rede Fibra. Ciênc Saúde Colet 2016; 21:3483-92.

60. Vieira LS, Gomes AP, Bierhals IO, FaríasAntúnez S, Ribeiro CG, Miranda VIA, et al. Falls among older adults in the South of Brazil: prevalence and determinants. Rev Saúde Pública 2018; 52:22.

61. Campos ACV, Ferreira EF, Vargas AMD. Determinantes do envelhecimento ativo segundo a qualidade de vida e gênero. Ciênc Saúde Colet 2015; 20:2221-37.

62. Ribeiro EE, Maia-Ribeiro EA, Brito E, Viegas K, Silva T, Mota KM, et al. Aspects of the health of Brazilian elderly living in a riverine municipality of Amazon rainforest. Revista Amazonense de Geriatria e Gerontologia 2013; 5:4-17.

63. Stamm B, Leite M, Hildebrandt LM, Kirchner RM, Menezes LP. Cair faz parte da vida: fatores de risco para quedas em idosos. Rev Pesqui Cuid Fundam 2016; 8:5080-6.

64. Cesar JA, Oliveira-Filho JA, Bess G, Cegielka R, Machado J, Gonçalves TS, et al. Perfil dos idosos residentes em dois municípios pobres das regiões Norte e Nordeste do Brasil: resultados de estudo transversal de base populacional. Cad Saúde Pública 2008; 24:1835-45.

65. Benedetti TRB, Binotto MA, Petroski EL, Gonçalves LHT. Atividade física e prevalência de quedas em idosos residentes no sul do Brasil. Rev Bras Geriatr Gerontol 2008; 11:14554.

66. Chehuen Neto JA, Brum IV, Braga NAC, Gomes GF, Tavares PL, Silva RTC, et al. Percepção sobre queda como fator determinante desse evento entre idosos residentes na comunidade. Geriatr Gerontol Aging 2017; 11:25-31. 
67. Sanchez-Garcia S, Garcia-Pena C, Salva A, Sanchez-Arenas R, Granados-Garcia V, Cuadros-Moreno J, et al. Frailty in communitydwelling older adults: association with adverse outcomes. Clin Interv Aging 2017; 12:100311.

68. Halil M, Ulger Z, Cankurtaran M, Shorbagi A, Yavuz BB, Dede D, et al. Falls and the elderly: is there any difference in the developing world? A cross-sectional study from Turkey. Arch Gerontol Geriatr 2006; 43:351-9.

69. Zimba Kalula S, Ferreira M, Swingler G, Badri M, Aihie Sayer A. Prevalence of falls in an urban community-dwelling older population of Cape Town, South Africa. J Nutr Health Aging 2015; 19:1024-31.

70. Gale CR, Cooper C, Aihie Sayer A. Prevalence and risk factors for falls in older men and women: the English Longitudinal Study of Ageing. Age Ageing 2016; 45:789-94.

71. Muir SW, Beauchet O, Montero-Odasso M, Annweiler C, Fantino B, Speechley M. Association of executive function impairment, history of falls and physical performance in older adults: a cross-sectional population-based study in eastern France. J Nutr Health Aging 2013; 17:661-5.

72. Martin KL, Blizzard L, Srikanth VK, Wood A, Thomson R, Sanders LM, et al. Cognitive function modifies the effect of physiological function on the risk of multiple falls: a population-based study. J Gerontol A Biol Sci Med Sci 2013; 68:1091-7.

73. Mesas AE, Lopez-Garcia E, Rodriguez-Artalejo F. Self-reported sleep duration and falls in older adults. J Sleep Res 2011; 20(1 Pt 1):21-7.

74. Diz JB, Leopoldino AA, Moreira BS, Henschke N, Dias RC, Pereira LS, et al. Prevalence of sarcopenia in older Brazilians: a systematic review and meta-analysis. Geriatr Gerontol Int 2017; 17:5-16.

75. Da Mata FA, Pereira PP, Andrade KR, Figueiredo AC, Silva MT, Pereira MG. Prevalence of frailty in Latin America and the Caribbean: a systematic review and meta-analysis. PLoS One 2016; 11:e0160019.

76. Rodrigues IG, Barros MBA. Osteoporosis selfreported in the elderly: a population-based survey in the city of Campinas, São Paulo, Brazil. Rev Bras Epidemiol 2016; 19:294-306.

77. Etman A, Wijlhuizen GJ, van Heuvelen MJ, Chorus A, Hopman-Rock M. Falls incidence underestimates the risk of fall-related injuries in older age groups: a comparison with the FARE (Falls risk by Exposure). Age Ageing 2012; 41:190-5.
78. Nicklett EJ, Lohman MC, Smith ML. Neighborhood environment and falls among community-dwelling older adults. Int J Environ Res Public Health 2017; 14:175.

79. Marengoni A, Angleman S, Melis R, Mangialasche F, Karp A, Garmen A, et al. Aging with multimorbidity: a systematic review of the literature. Ageing Res Rev 2011; 10:430-9.

80. Sanchez-Ramirez DC, van der Leeden M, Knol DL, van der Esch M, Roorda LD, Verschueren S, et al. Association of postural control with muscle strength, proprioception, self-reported knee instability and activity limitations in patients with knee osteoarthritis. J Rehabil Med 2013; 45:192-7.

81. Handrigan GA, Maltais N, Gagne M, Lamontagne P, Hamel D, Teasdale N, et al. Sex-specific association between obesity and self-reported falls and injuries among communitydwelling Canadians aged 65 years and older. Osteoporos Int 2017; 28:483-94.

82. Yang Y, Hu X, Zhang Q, Zou R. Diabetes mellitus and risk of falls in older adults: a systematic review and meta-analysis. Age Ageing 2016; 45:761-7.

83. Marin R, Diaz M. Estrogen interactions with lipid rafts related to neuroprotection. Impact of brain ageing and menopause. Front Neurosci 2018; 12:128.

84. Yu PL, Qin ZH, Shi J, Zhang J, Xin MZ, Wu ZL, et al. Prevalence and related factors of falls among the elderly in an urban community of Beijing. Biomed Environ Sci 2009; 22:179-87.

85. Iser BPM, Stopa SR, Chueiri PS, Szwarcwald CL, Malta DC, Monteiro HOC, et al. Prevalência de diabetes autorreferido no Brasil: resultados da Pesquisa Nacional de Saúde 2013. Epidemiol Serv Saúde 2015; 24:305-14.

86. Hewston P, Deshpande N. Falls and balance impairments in older adults with type 2 diabetes: thinking beyond diabetic peripheral neuropathy. Can J Diabetes 2016; 40:6-9.

87. Landi F, Liperoti R, Russo A, Giovannini S, Tosato M, Capoluongo E, et al. Sarcopenia as a risk factor for falls in elderly individuals: results from the ilSIRENTE study. Clin Nutr 2012; 31:652-8.

88. Siqueira FV, Facchini LA, Hallal PC. The burden of fractures in Brazil: a population-based study. Bone 2005; 37:261-6.

89. Oliveira AS, Trevizan PF, Bestetti MLT, Melo RC. Fatores ambientais e risco de quedas em idosos: revisão sistemática. Rev Bras Geriatr Gerontol 2014; 17:637-45.

90. Tinetti ME, Kumar C. The patient who falls: "it's always a trade-off”. JAMA 2010; 303:258-66. 


\section{Resumo}

As quedas em idosos resultam em uma enorme carga epidemiológica, clínica e econômica nesta faixa etária no mundo inteiro, com alto risco de incapacitação grave. O estudo teve como objetivo estimar a prevalência de quedas e fatores associados entre idosos brasileiros, através de uma revisão sistemática com meta-análise. Foram realizadas buscas nas bases de dados SciELO, PubMed, LILACS, Web of Science, Scopus e PsycINFO, sem restrição de idioma ou ano de publicação. Foram incluídos estudos sobre idosos de ambos os sexos com 60 anos ou mais, residindo na comunidade, com amostras de $\geq 300$ participantes em cada estudo. Os critérios de exclusão foram estudos realizados especificamente em idosos diagnosticados com doenças crônicas incapacitantes que predispõem a quedas. $O$ risco de viés dos estudos incluídos foi avaliado com uma ferramenta de avaliação crítica focada em desenhos de prevalência. Foi utilizada uma meta-análise de efeitos aleatórios para combinar as prevalências de quedas entre estudos. A análise exploratória foi realizada pela investigação das estimativas de subgrupos, razões de prevalência e meta-regressão. Foram incluídos 37 estudos, com um total de 58.597 participantes. A prevalência de quedas nos últimos 12 meses foi de 27\% (IC95\%: 24,3-30,0), com estimativas significativamente mais altas em mulheres ( $R P=1,57$; IC95\%: 1,321,86), na faixa etária $\geq 80$ anos comparado com 60-69 anos ( $R P=1,46$; IC95\%: 1,15-1,84) e em idosos da Região Centro-oeste, comparado com os da Região Sul ( $R P=1,36$; IC95\%: 1,10-1,69). O tamanho do risco de viés não impactou a heterogeneidade na meta-análise de 12 meses. Essas estimativas apoiam fortemente as intervenções públicas baseadas em evidências para prevenir quedas em idosos, especialmente nas mulheres e nos idosos mais velhos.

Acidentes por Quedas; Idoso; Prevalência

\section{Resumen}

En todo el mundo, las caídas representan una carga grande epidemiológica, clínica, y económicamente en la población mayor, presentando altas tasas de discapacidad severa. El objetivo de este estudio fue estimar la prevalencia de las caídas $y$ los factores asociados en ancianos brasileños, usando una revisión sistemática con metaanálisis. Las búsquedas se realizaron en bases de datos como: SciELO, PubMed, LILACS, Web of Science, Scopus y PsycINFO sin restricciones de fecha o lengua. Se incluyeron estudios sobre ancianos residentes en comunidades con una edad $\geq 60$ años de ambos sexos y con un tamaño de la muestra de $\geq 300$ participantes. Los criterios de exclusión fueron estudios dirigidos específicamente a adultos mayores, diagnosticados con enfermedades crónicas incapacitantes que les predisponen a caídas. El riesgo de sesgo en los estudios incluidos fue evaluado usando una herramienta de evaluación crítica, centrándose en los diseños de prevalencia. Se utilizó un metaanálisis de efectos aleatorios para agrupar la prevalencia de caídas a través de estos estudios. Se realizó un análisis exploratorio, examinando estimaciones de subgrupos, tasas de prevalencia y meta-regresión. Se incluyeron treinta y siete estudios, implicando a 58.597 participantes. En doce meses la prevalencia de caídas fue $27 \%$ (IC95\%: 24,3-30,0), con estimaciones significativamente más altas en mujeres que en hombres ( $P R$ = 1,57; IC95\%: 1,32-1,86), en el grupo de edad $\geq$ 80 años, en comparación con el grupo de edad de 60-69 años ( $P R=1,46$; IC95\%: 1,15-1,84), y en participantes procedentes de la región centro-oeste, respecto a los participantes de la región sur $(P R=$ 1,36; IC95\%: 1,10-1,69) de Brasil. El riesgo de sesgo en los resultados no impactó la heterogeneidad en el metaanálisis de 12 meses. Estas estimaciones apoyan fuertemente las intervenciones públicas basadas en evidencias para prevenir caídas en ancianos brasileños, especialmente en mujeres y en la población más anciana.

Accidentes por Caídas; Anciano; Prevalencia
Submitted on 14/Jun/2018

Final version resubmitted on 12/Mar/2019

Approved on 29/Apr/2019 